\title{
PHILOSOPHIE \\ ET ÉPISTÉMOLOGIE
}

Antoinette VIRIEUX-REYMOND, Les Grandes étapes de l'épistémologie jusqu'd Kant. Préf. de Gérard LE COAT. Genève, Patiño, 1986. $14 \times 20,5,138$ p., index, bibliogr.

Docteur ès lettres en Sorbonne, ancien privat-docent à l'Université de Lausanne et professeur au Gymnase Vinet, $\mathbf{M}^{\mathrm{me}} \mathrm{A}$. Virieux-Reymond est l'une des plus éminentes épistémologues de langue française ; on connaît, depuis longtemps, avec faveur, sa belle thèse sur La Logique et l'épistémologie des stoïciens (1949), ses pénétrants ouvrages sur La Logique formelle, Platon ou la géométrisation de l'univers, Arnold Reymond, Pierre-Maxime Schuhl, Introduction à l'épistémologie et ses nombreux articles de revues ou communications de congrès. Voici que les Éditions Patiño nous proposent aujourd'hui une précieuse esquisse, tracée par notre collègue, de l'élaboration des idées scientifiques occidentales, depuis les Grecs jusqu'à Kant exclu, soit pendant vingt-trois siècles très riches en spéculations et découvertes. Ce nouveau livre comble une grave lacune, puisque nous possédions seulement, dans l'aire culturelle francophone, le volume de Raymond Bayer, Épistémologie et logique depuis Kant, jusqu'à nos jours (1954). Comme l'observe le préfacier (p. IX), l'original compendium de notre collègue de Lausanne constitue « l'aboutissement logique de plus de trente années de questionnement ", tant en Suisse romande qu'aux Semaines de synthèse de Paris et aux diverses rencontres des sociétés de philosophie de langue française.

Grâce à ce raccourci fort soigneusement établi, le lecteur suit l'effort épistémologique trimillénaire, dans ses principales phases. D'abord, dans l'Antiquité (p. 5-38), à travers les présocratiques (surtout Zénon d'Elée, montrant que « le temps n'est pas une somme d'instants ", p. 8), les hippocratiques, Thucydide (promoteur lointain de l'histoire scientifique), Platon (dénonçant les obstacles épistémologiques et inaugurant la mathématisation de l'univers), Aristote (réagissant là contre et insistant sur le concret), Théophraste, Épicure et les Stoïciens (instaurant les « notions communes " et fondant les " jugements vrais " sur le logos, enfin les sceptiques. $\mathbf{M}^{\mathrm{mc}} \mathrm{A}$. Virieux-Reymond inventorie ensuite le Moyen 
Âge (p. 39-60), dont l'apport est bien plus grand et valable qu'on ne le croit généralement ; à la suite de Duhem et d'autres, on s'aperçoit de la sorte - au-delà même de l'alchimie arabe - que la réflexion critique sur les méthodes et principes des sciences a été poussée assez loin par les Médiévaux : entre autres, chez Pierre l'Espagnol ; dans l'École de Chartres, platonicienne et mathématisante ; chez Robert Grosseteste (p. 48-54), combinant le mathématisme avec l'expérimentation ; chez Roger Bacon ; chez Bradwardine (auteur du Traité des proportions), qui amorce « le travail d'abstraction qui devait permettre la naissance de l'algèbre " (p. 57) ; chez les deux Buridan (théorie de l'impetus), etc. À la Renaissance (p. 64-82), les points de vue s'élargissent davantage avec Nicolas de Cues (le principe de continuité et la pesée), Nifo, Copernic, Viète, Bombelli (les nombres complexes), Galilée et Francis Bacon. L'époque classique est plus riche encore (p. 83-114) : Descartes qui "renoue avec le rêve des géométrisations du réel ", p. 89, et qui fait rentrer dans ce cadre les phénomènes mouvants comme les réalités statiques ; Gassendi qui, bien que trop sensualiste, " ressuscite la physique démocritéenne et donne à la science moderne la base ontologique qui lui manquait », p. 91 ; Pascal, " complémentaire du cartésianisme "; Newton, introducteur de la pensée corpusculaire, de la méthode excluant les grandes hypothèses, de la physique rénovée et mathématique, dépassant l'atomisme ; Rohault, recourant aux inobservables ; Malebranche ; enfin, Leibniz, précurseur de la logistique, restaurateur de la notion de force, législateur des principes et défenseur d'un cartésianisme réformé ; son épistémologie " est unie étroitement à une métaphysique, où toutes les philosophies du passé ont déposé ce qu'elles avaient gardé de vivant $»$, p. 124.

En résumé, le survol de ces trois millénaires révèle une perpétuelle oscillation entre l'unité et le pluralisme des doctrines scientifiques, ainsi que le progrès toujours plus grand de l'expérimentalisme ; en outre, on y voit qu' « il n'y a pas eu une seule Renaissance, mais une série de renaissances, qui se sont succédé et qui coïncident toutes avec la redécouverte de nouveaux textes de l'Antiquité grecque, confrontés avec des expériences originales par les commentateurs ", p. 119. À notre avis, le vademecum proposé par A. Virieux-Reymond rendra les plus grands services, tant aux spécialistes qu'au public cultivé.

Alain GuY.

HÉraClite, Fragments. Texte établi, traduit, commenté par Marcel CONCHE. Paris, P.U.F., 1986. $15 \times 21,5,496$ p., bibliogr., concordance, index des citateurs, index des mots grecs, index des passages d'auteurs anciens, 5 fig. (" Épiméthée »).

Les principes de cette édition marquante, dont le succès de librairie n'a pas attendu les recensions de spécialistes, puisqu'une deuxième édition a déjà suivi la première (sans autre changement que des corrections mineures), sont clairement exposés en Introduction. D'abord, il s'agit d'une édition des Fragments 
(et non de " fragments et témoignages »), c'est-à-dire d'un travail qui se concentre, autant qu'il est possible, sur les reliquia de la lettre même d'Héraclite, avec la conviction qu'ils " ont incomparablement plus d'importance que les maigres données de la doxographie " (p. 5), source, par surcroît, de contaminations (cf. p. 6). En second lieu, la célèbre réputation d'obscurité du penseur doit être appréciée : " la rapide diffusion de l'héraclitéisme dans le monde grec " (p. 8) n'a pu se faire que dans des conditions assez ingrates (non-séparation des mots, aucune ponctuation, etc.) qui n'impliquent pas une volonté d'obscurité à l'origine. Il y a la de quoi encourager le travail d'interprétation, tout comme ce fait que « le nombre des fragments originaux reste du même ordre depuis plus d'un siècle " (p. 10). Enfin et surtout est affirmé un grand principe d'ordre, à partir d'une expérience d'interprète qui voit dans l'ensemble des fragments « un tout harmonieux, d'une admirable cohérence " (p. 13). Ce principe d'ordre ne prend pas en considération l'impossible restitution de la disposition des fragments dans le livre perdu (que l'on retrouvera peut-être un jour, est-il dit, p. 8) ; il est méthodologique et reproduit la marche même de l'élucidation ab facilioribus ad difficiliora (cf. p. 13-14), selon un progrès tout à la fois " profondément rationnel " (p. 14) et conforme au déroulement essentiel des étapes effectives de cette élucidation.

Dès l'Introduction est soulignée une idée-mère, l'idée-mère de l'héraclitéisme : « ce qui est dit, et qui nous est montré, est la réalité dans son ensemble, telle qu'elle est maintenant et sera toujours, dans son immuable mouvance, car il n'est rien à la fois de plus permanent et de plus changeant que le monde " (p. 13). Les premiers fragments abordés sont ceux qui concernent le Logos et l'unité de toutes choses (Diels B 50 et B 1) ; l'unité de tout est l'unité des contraires, bien distincte d'une prétendue unité des contradictoires, " car ceux-ci sont, comme tels, exclusifs de l'unité » (p. 27) et marquent la séparation du vrai et du faux. Le logos, le discours vrai « n'est pas un avec son contradictoire [...] ne fait pas partie du tout : il est hors du tout, justement pour pouvoir dire, dévoiler le tout " (ibid.). Cette idée de la vérité du tout échappant à la loi de l'unité des contraires pour en être l'expression revient inlassablement dans la suite du commentaire (cf. p. 28, 36, 151, 238-240, 469...). Elle s'accompagne d'une autre séparation, celle du philosophe, porteur du discours vrai, et des autres hommes (cf. p. 36, $47 .$. ), fâcheuse celle-là, puisqu'elle isole le philosophe et coupe les autres hommes de la loi du réel en les cantonnant dans la particularité de leurs désirs et l'étroitesse des relations sujet-objet. Ce que fonde au contraire Héraclite, c'est « une nouvelle maîtrise sur les choses et sur eux-mêmes [les hommes], une nouvelle stratégie de la vie sensée, maîtrise fondée sur la connaissance des choses telles qu'elles ont été, sont et seront toujours (toujours semblables quoique toujours autres), nullement, comme celle issue de l'art de la divination ou de la prévision scientifique moderne, sur la connaissance de l'avenir " (p. 39, cf. p. 52-54). De même, plus bas, à propos des fragments Diels B 73 et 75 : " si nous agissons en ayant conscience, au niveau du langage, de l'unité du positif et du négatif, nous intégrons le négatif dans notre stratégie et le maîtrisons " (p. 69, cf. p. 72-73, B 71 ainsi que p. 83).

Le fondement même tant de l'action bien éveillée que de la théorie est ainsi 
l'unité des opposés, positif-négatif, semblable-différent, ou encore (comme il a été dit au début) immuable et changeant. Sur cette « intuition fondamentale " (p. 108) se greffe la critique de principe qui vise divers penseurs contemporains, notamment Pythagore de Samos et Xénophane : « il y a chance, écrit M. Conche, pour que ce qui, à ses yeux, leur a manqué, ce soit d'avoir vu, dans le réel, l'unité des contraires, l'intelligence consistant à saisir partout cette unité » (p. 93, cf. $B 40$ et 129). De même Homère, Hésiode, Archiloque (B 42, 56, 57). On ne saisit pas le sens précis des attaques d'Héraclite si l'on s'arrête par exemple au reproche de disparate adressé à Pythagore ou à l'exclusion d'Homère souhaitée par respect de l'esprit agonistique de la culture et de l'éducation grecques. Car le commentateur note, comme il convient, l'effort d'unification et de systématisation de Pythagore (et aussi bien, dans une autre direction, d'Hésiode, cf. p. 91) ou l'imprégnation de l'esprit agonistique chez Homère (cf. p. 117), mais il souligne l'échec de ces tentatives qui ne reconnaissent pas " le caractère universel et nécessaire " (p. 93) de l'unité des contraires (Pythagore, aussi Xénophane) et entre autres la nécessité de la joute homérique (cf. p. 117). Au contraire, « Héraclite [...] est en accord profond avec l'esprit de l' $\alpha \gamma \omega v$ " (ibid).

Beaucoup d'interprètes ont attribué complaisamment une valeur héraclitéenne au fameux fragment B 93 qui dit que « le maître dont l'oracle est celui de Delphes ne dit ni ne cache mais donne des signes " (tiré de Plutarque, Oracles de la Pythie, 404 d). Mais il n'y a pas plus de raisons d'y trouver une affinité avec l'héraclitéisme que, par exemple, de trouver un sens spinoziste à la métaphore des deux chiens, l'animal et la constellation. L'oracle de Delphes reste dans le vague du clair-obscur, tandis que le Logos du philosophe dit clairement et inébranlablement la vérité : Héraclite n'hésite pas à entrer en lutte, en rivalité avec Apollon et il le dépasse (cf. p. 153). Tous les fragments portant sur les mythes et croyances (B 14, 15,92...) doivent être compris dans le même sens. En particulier, l'espoir en une vie future que promettent les mystères de Déméter ou ceux de l'orphisme va à l'encontre de «l'ordre de la nature, ordre éternel et divin " (p. 166), c'est donc un espoir aussi impie que vain. « Les dogmes et les rites des religions sont à rejeter entièrement, car il ne sert à rien d'errer dans la nuit. Le logos est discours de la seule raison, libre à l'égard de tous les délires traditionnels » (p. 170 et B 14 a). Toutefois, les philosophes étant rares (cf. B 69 et p. 180-181), Héraclite a probablement admis la valeur thérapeutique de certains rites dionysiaques : “ les obscénités libèrent de l'obsession » (p. 183), chez les gens ordinaires, chez la multitude.

Les fragments qui insistent sur la mesure sont à lire, eux aussi, selon le principe du discours vrai, la stable loi du changement posée dès le début (cf. p. 35) ; non pas que le logos gouverne le monde, il est plutôt la vérité qui se constitue en face de lui et, on l'a vu, hors de sa totalité, corrélativement à sa rationalité immanente. Héraclite semble s'inspirer de la tradition milésienne et plus particulièrement de la justice cosmologique d'Anaximandre (cf. p. 194). Les fragments astronomiques sont lumineusement expliqués par le commentateur, notamment B 120, sur lequel Zeller avouait son embarras, et B 3, élucidé grâce au rapprochement de l'apparence du Soleil et du thème héraclitéen du contraste qualitéquantité (cf. p. 98 et plus haut, p. 95, B 22). La « grande année » de 10800 ans 
( 360 fois trente ans ou une génération) n'a pas le sens astronomique d'un retour des conjonctions planétaires, ni le sens cosmique d'un embrasement périodique total (l'ekpyrosis des Stoïciens, cf. p. 203, 286, 301 et déjà p. 6), ni non plus le sens anthropologique d'un retour éternel du même chez les individus, mais bien plutôt un sens météorologique (cf. p. 204-205), avec un « grand hiver " et un " grand été ", sans déluge ni embrasement universels, qui apporteraient la démesure contraire à la loi du monde, en réduisant, par impossible, l'unité des contraires à un seul des deux.

Il faudrait encore, pour donner quelque idée de l'unité, de la richesse et de l'immense érudition de toutes ces analyses, évoquer le commentaire des fragments relatifs à la vie politique $(\mathrm{B} 114,44,33)$, à la cosmologie des éléments et à la théorie de l'âme (B 90,31 , etc.). On se contentera de souligner ici la présence constante tout au long du volume du principe d' "immuable mouvance " et d'unité des contraires qui s'exprime dans la vérité séparée dont le porteur est le philosophe. C'est ce que rappelle une dernière fois le commentaire du fameux : "Tout s'écoule " (le logos " est hors du tout ", p. 469, cf. p. 28, etc., déjà cité ; sur le fragment tiré de Simplicius, déjà des éléments de commentaire p. 11 et 13 : la fin rejoint le commencement). Ainsi se dessinent avec fermeté les traits caractéristiques de la pensée d'Héraclite : le monde est incréé, éternel et éternellement changeant sous la loi immanente de l'unité réglée des contraires, loi transparente du tragique de l'existence, perçue au moins de quelques-uns et susceptible de leur éviter la démesure, ainsi qu'aux cités qui les reconnaissent pour guides ; sont niés les dieux et toutes les croyances populaires, tout comme la vie post mortem, seul est divin le cosmos en son organisation immanente « où tout est relié à tout et partout hors de soi - puisque l' "être"' est relation " (p. 53), ainsi que peut-être le philosophe (p. 78-79).

Risquons, en terminant la recension de cet ouvrage exceptionnel par l'ampleur de son information comme par la clarté et la vigueur de ses thèses, l'esquisse de deux objections.

D'abord, je ne me sens pas absolument convaincu par l'élimination des termes contradictoires in rebus (cf. p. 27). Est-elle compatible avec le maintien du négatif parmi les termes opposés (notamment p. 28, 73) ? Sans doute, en B 62 (p. 369-371), le maniement de la dialectique est critique, non pas ontologique, en concordance avec l'ensemble des fragments antireligieux. Il n'en va pas de même, toutefois, pour B 126, étudié à la suite, ni pour B 67 (cf. p. 377 sqq.). Car si la négation d'un terme implique l'affirmation de l'autre non moins que l'affirmation de celui-ci la négation du premier, il me semble que l'on sort de l'opposition de contrariété et que la réciprocité des deux termes, sans tiers possible, est une relation de contradiction. " Dans la réalité aussi, l'un des deux (c'està-dire contraires) ne se conçoit pas sans l'autre " (p. 378) et tout ce qui n'est pas froid est chaud, tout ce qui est humide s'oppose au sec, sans tiers possible et le passage s'opère de l'un à l'autre dans les deux sens (sans privilège, du reste, pour " le travail du négatif ") selon les transmutations du devenir. D'autre part, Conche utilise pour éliminer les contradictoires de la réalité les distinctions classiques d'Aristote. Mais précisément, si Aristote distingue dans les Catégories (cf. p. 25-27) les deux types d'opposition, il est remarquable qu'il dynamise, en quelque 
sorte, les contradictoires (cf. p. 26 et 27) en leur conférant l'activité des verbes à l'infinitif, ce qui ne recouvre pas exclusivement l'activité du jugement, mais aussi bien, chez Héraclite, celle du mouvement des opposés dans un monde sans réification d'êtres substantiels (sans compter l'emploi en B 66, de l'idée de jugement à propos du « pouvoir du feu », p. 301 et plus généralement de la loi de " justice " immanente). De plus, il semble bien qu'en étant très réticent en face d'Héraclite, Aristote croie à une affirmation des contradictoires chez un penseur qui, d'après des on-dit, aurait conçu « que la même chose est et n'est pas ", mais, ajoute-t-il, « tout ce qu'on dit, on n'est pas obligé de le penser » (Métaph. Gamma 3, 1005 b 23-25, trad. Tricot). Or, le maître d'Aristote qui, le premier, a fait traiter par un de ses personnages, le médecin Eryximaque dans le Banquet, le prestigieux Héraclite de maladroit incapable d'exprimer ses idées (187 ab, avec allusion à B 51), s'est, comme on sait, intéressé jusqu'à la fascination au penseur d'Ephèse (notamment dans le Sophiste) et l'on ne comprend pas très bien, ce qui n'est d'ailleurs qu'un argument trop extérieur pour être décisif, comment rendre compte de « l'entrelacement » des Idées dans la dernière philosophie de Platon si " les muses d'Ionie », si Héraclite n'avait pas admis un certain jeu de contradictoires dans la réalité des choses.

Une seconde objection porterait sur la transcendance du Logos. Le commentateur distingue (p. 238-240, à propos de B 108, " la sagesse séparée de tout ») deux sens de la transcendance, le premier signifiant seulement qu'une vérité domine les faits et n'en dépend pas, le second qu'un être domine, non plus en vérité, mais effectivement et réellement, les autres êtres. Il faut interpréter Héraclite dans le premier sens, celui de la connaissance, c'est évident. Mais ce « savoir de la totalité » (p. 239) doit nécessairement lui être extérieur : le Logos ne serait-il pas, si l'on se contente du premier sens de la transcendance, en contradiction avec le Tout, puisqu'il n'en est pas une partie ? Suffit-il d'affirmer la spécificité du Logos, la connaissance visant le réel sans en faire partie comme telle, pour se délivrer de la question du statut réel de cette transcendance et des conditions de possibilité de sa séparation? On comprend en tout cas que la tentation est grande de passer du premier sens de la transcendance au second, pour réintégrer le savoir dans une hiérarchie ontologique, contraire à l'esprit héraclitéen (Diels faisait d'Héraclite, cf. p. 11, " le fondateur de l'Idéalisme en philosophie »). Il vaudrait mieux en effet, semble-t-il, s'accommoder d'une contradiction dans l'être entre le tout et sa connaissance comme partie détachée, par où serait respecté le naturalisme immanentiste de la doctrine.

On attend avec le plus grand intérêt l' « exposé synthétique du système » annoncé en fin d'Introduction (p. 14). 
AVICENNE, Le Livre de science. I Logique, métaphysique; II Science naturelle, mathématiques. Trad. par Mohammad ACHENA et Henri MASSÉ, $2^{c}$ éd. revue et corrigée par Mohammad ACHENA. Paris, "Les Belles Lettres »/UNESCO, 1986. 12,5 × 19,5, 260 p. (« Coll. UNESCO d'œuvres représentatives/série persane $")$.

La première édition de cet ouvrage a paru en deux volumes datés respectivement de 1955 et 1958 . Les changements apportés par la seconde affectent la traduction ( " certains paragraphes et même certaines pages sont complètement modifiés », écrit M. Achena, I, p. 5) ; l'Introduction générale, qui avec la liste des ouvrages cités occupe maintenant quarante pages (I, p. 7-47) contre quinze dans la première édition (cette Introduction comprend une biographie d'Avicenne et des notices sur sept de ses principaux ouvrages) ; et les notes du premier tome, qui occupent vingt pages (p. 259-280) contre une dizaine dans la première édition ; dans le second tome, les figures sont maintenant à leur place dans le texte au lieu d'être rejetées toutes à la fin.

Le Livre de science a été écrit en persan (Däneš-Näme), c'est la seule dans ce cas parmi les encyclopédies d'Avicenne (qui, plus ou moins longues, sont au nombre de six). Elle comporte, d'après la Préface de l'auteur, cinq parties : logique, science de la nature, astronomie, musique, métaphysique (I, p. 63-64). Mais le contenu peut s'en analyser autrement : la logique, et les trois sciences théorétiques telles que les énumère Aristote ; ou encore, logique, métaphysique, physique, et quadrivium. Quelques remarques sur ces points : 1) cette dernière partie a été rédigée en persan par Ğawzḡāni (qui fut aussi le biographe d'Avicenne) d'après plusieurs écrits du maître, la rédaction originale s'étant perdue (voir ce qu'il en dit : II, p. $91-92$; et ce qu'en dit M. Achena : II, p. 7-9) ; 2) l'ordre suivi est particulier : géométrie, astronomie, arithmétique, musique ; du moins la répartition de ces quatre sciences en deux couples - science des figures, sciences du nombre - est conforme à la tradition néo-pythagoricienne. Quant aux trois sciences traitées les premières, et de loin le plus longuement, la séquence logiquemétaphysique-physique est, dit lui-même Avicenne (I, p. 64), " contraire aux usages et à la coutume ". De fait, dans toutes ses autres encyclopédies, il suit l'ordre logique-physique-métaphysique (qu'on trouve déjà dans les catalogues alexandrins des œuvres d'Aristote). La raison de cette nouvelle répartition apparaît en I, p. 118 : le sujet d'une science, c'est-à-dire « ce dont elle examine l'état ", est le plus souvent établi dans une autre science ; or la métaphysique, qui est la " science de l'être absolu en tant qu'absolu » et des « causes qui concernent tout l'être " (I, p. 134-135), doit de ce fait précéder la physique, qui traite d'une sorte particulière de l'être : celui dont les états ne peuvent se concevoir sans la matière (II, p. 13). On notera que Šahrastāni, qui dans son Livre des religions et des sectes expose la philosophie d'Avicenne en aboutant des extraits de la Nă̌àt, suit le même ordre que ce Livre de science alors que la Nağăt suit l'ordre logique-physique-métaphysique. Plus important, les Intentions des philosophes, de Gazälī, écrit une soixantaine d'années après Le Livre de science, suit le même ordre que ce dernier : voir là-dessus Manuel Alonso Alonso, Algazel. Maqāsid al-Faläsifa. Intenciones de los Filosofos, Barcelone, 1963, p. XLV- 
LII ; et Jules Janssens, "Le Dânesh-Nâmeh d'Ibn Sînâ : un texte à revoir ? ", Bulletin de philosophie médiévale, 28, 1986, p. 163-177 (168-175 ; cet article compare en outre le Däneš-Näme avec les Gloses d'Avicenne sur Aristote : p. 164-168). Mais il est excessif de dire (I, p. 44) que les Intentions des philosophes soient « la traduction littérale " du Livre de science.

Quant au contenu de cette encyclopédie, il ne peut être question de le résumer, pas plus que de présenter la situation de la philosophie d'Avicenne par rapport à celle d'Aristote - fidélité apparente, importantes modifications en profondeur ; on se contentera donc de quelques remarques. Et d'abord, celle-ci : Le Livre de science est la seule encyclopédie avicennienne dont on ait la traduction complète en français (avec celle des Išărät, certes, procurée en 1951 par Amélie-Marie Goichon, mais cette œuvre est d'un esprit tout particulier et d'une lecture ardue) ; nous avons donc là le passage obligé, pour tout lecteur en cette langue, vers une connaissance complète de la philosophie d'Avicenne. On ne peut donc que se féliciter que l'éditeur en donne une réédition améliorée. Signalons maintenant quelques points. Dans la Logique (tome I), on notera une invention d'Avicenne, du moins revendiquée comme telle : celle des syllogismes conjonctifs (« par connexion ») composés d'une prémisse hypothétique ( conditionnelle conjonctive ») et d'une prémisse catégorique (" attributive ") : voir I, p. 102-103 ; Ṭusî, commentateur d'Avicenne, le confirme (voir l'Introduction à la Logique, l, p. 51) ; Averroès niera l'originalité d'Avicenne sur ce point. P. 103-108, critique des méthodes dialectiques suivies par les juristes et les théologiens : c'est l'un des innombrables épisodes de la guerre entre la philosophie et les sciences islamiques traditionnelles. Dans la Métaphysique (tome I), les pages 156-163 exposent, avec une concision qui exige beaucoup du lecteur, une doctrine pour laquelle Avicenne occupe une place capitale dans l'histoire de l'ontologie : celle des rapports entre l'existence et la quiddité ; mais on notera l'absence en ces développements du concept de " chose », utilisé ailleurs par Avicenne pour désigner une structure essentielle indifférente à l'existence (la note $2 ;$ p. 271-272, confirme implicitement cet abandon). Or ce concept s'était formé antérieurement au cours de controverses théologiques, Avicenne l'a assimilé à sa philosophie : la guerre évoquée tantôt n'allait pas sans annexions, de part et d'autre d'ailleurs. Dans la Physique (tome II) : le mouvement selon la position (p. 17, 19) est, Avicenne le dit ailleurs, ajouté par lui à la liste aristotélicienne des quatre sortes de changement (selon la substance, la quantité, la qualité, le lieu) ; il lui sert à rendre compte du mouvement circulaire et notamment de celui du premier Ciel, qui est sans lieu. On notera aussi l'évocation d'expériences très concrètes (p. 34-35, la pointe de la flèche qui fond ; p. 40-41, description d'un phénomène atmosphérique observé par Avicenne en un lieu qu'il nomme). Signalons enfin que cette Physique contient aussi des allusions à divers traités du corpus aristotélicien, tels les Météores.

Il reste à dire que les diverses introductions et les notes de ces deux tomes apportent, sans être exagérément développées, des renseignements précieux au lecteur : soit factuels (biographie, sort des œuvres ; voir aussi, I, p. 38, une notation intéressante sur la situation des Išărăt dans l'histoire spirituelle du monde islamique) ; soit conceptuels. On fera deux remarques critiques : 1) le mot tagawhur, " substantification " selon la traduction d'Henry Corbin (I, p. 37), n'est pas de 
l'invention d'Avicenne : on le trouve déjà chez Färäbi ; 2) la traduction de l'autobiographie d'Avicenne, écrite en arabe et transmise par Ibn Abi Ușaybica, conserve un contre-sens de la première édition. Avicenne n'a pas écrit : "je m'appliquai à lire et à étudier les Fușüs al-ḥikam [de Färäbi] et d'autres commentaires sur la physique et la métaphysique " (I, p. 13 ; les crochets sont dans le texte imprimé), mais bien : « des livres de physique et de métaphysique, textes et commentaires " (min al-fușüs wa-l-šurüh). Il y a déjà longtemps d'ailleurs que Kh. Georr et S. Pines ont contesté chacun de son côté, avec de forts arguments de critique interne et externe, l'authenticité farabienne des Fuṣus al-hikam ; l'enjeu est important, car si cet opuscule était de Färäbi, c'est à celui-ci qu'il faudrait attribuer le principal peut-être de l'ontologie avicennienne.

Jean JOLIVET.

Pierre-Simon Laplace, Essai philosophique sur les probabilités. Préf. René THOM, Postf. Bernard BRU. Texte de la $5^{e}$ éd., 1825. En appendice trois extraits des Mémoires de l'auteur. Paris, Christian Bourgois, 1986. 12,8 $\times$ $19,8,314$ p. (" Épistémè »).

La réédition de l'Essai philosophique sur les probabilités de Laplace sera sûrement pour beaucoup de lecteurs une occasion de prendre connaissance de ce que B. Bru, dans sa Postface, appelle " un des rares textes scientifiques qui puisse être considéré comme un classique de la littérature française ". Texte qu'à vrai dire bien peu, de nos jours, connaissent dans son intégralité.

Sa relecture se montrera passionnante à bien des égards pour un lecteur contemporain. Il y trouvera bien d'autres choses que la célèbre définition du déterminisme par laquelle il s'ouvre, et que chacun connaît (« Tous les événements, ceux-mêmes qui par leur petiiesse semblent ne pas tenir aux grandes lois de la nature, en sont une suite aussi nécessaire que les révolutions du soleil. ") : à côté de réflexions proprement philosophiques sur les fondements du calcul des probabilités, un précieux tableau d'ensemble des applications de celui-ci autour de 1800 , une réflexion profonde sur les conditions de la découverte scientifique, et des vues remarquables, que nous dirions prospectives, sur des problématiques scientifiques alors à peine ébauchées, mais appelées à de grands et féconds développements dans les siècles suivants.

Pour cette lecture ou cette relecture, la Postface de B. Bru sera un guide précieux. Il y avait peu de spécialistes français de l'histoire du calcul des probabilités ; d'où résultait que les contributions des savants français des XVIII ${ }^{e}$ et $\mathrm{XIX}^{e}$ siècles à son développement étaient ou méconnues, ou sous-estimées. Que Bernard Bru, déjà connu pour son édition critique du volume I des œuvres de Cournot, s'affirme décidément comme un spécialiste de haut niveau de l'histoire du calcul des probabilités devrait contribuer à remédier à cette distorsion.

En ce qui concerne les fondements, Laplace n'innove pas vraiment ; il reprend, en les approfondissant, en particulier les thèses des Bernoulli. Son apport per- 
sonnel tient surtout à ses propres travaux scientifiques sur la probabilité des causes (indépendants, semble-t-il, de ceux de Bayes). Son mérite, en général, et il est assez rare en cette matière, est d'être nuancé. Parti, dans son mémoire de 1773 (dont un extrait est publié dans le même volume), d'une conception subjectiviste à laquelle nos contemporains (de Finetti, Savage) n'ont apporté que des compléments techniques de portée somme toute limitée, et surtout beaucoup de dogmatisme, il évolue progressivement vers des idées faisant sa part à une certaine objectivité du hasard, comme phénomène affectant de grandes multiplicités d'objets. Comme le note B. Bru, p. 255 :

«Laplace ne s'embarrasse pas trop dans les nuances, puisque ces mots sont ambigus, on leur conservera leur ambiguïté. Le hasard commence par n'avoir "aucune réalité en lui-même", il ne désigne que "l'ignorance où nous sormmes des véritables causes", c'est le hasard stö̈cien à la mode au XviII siècle. Quelques années plus tard le hasard devient "l'ensemble des causes variables et inconnues qui rendent incertaine et irrégulière la marche des événements", c'est-à-dire les "véritables causes" elles-mêmes lorsqu'elles sont cachées. "

Parmi les idées auxquelles Laplace tient particulièrement figure celle qui définit la probabilité comme " rapport du nombre des cas favorables au nombre total des cas possibles ». Cette définition est aujourd'hui tenue généralement pour un fossile de l'histoire du calcul des probabilités. On peut cependant remarquer que, prise par un autre biais, elle fonde la probabilité comme un produit des symétries de l'épreuve aléatoire à laquelle elle s'applique, et que, dans certains cas, cette symétrie est tout à fait objective (cf. l'éclosion de la probabilité en théorie des Jeux, par application du théorème du minimax). En tout cas, c'est bien ainsi que le hasard se présente en mécanique quantique, comme produit de la symétrie des interactions quantiques (dans le cadre élargi de la théorie des Groupes).

Le tableau des applications, qui occupe une moitié à peu près de l'Essai, présente un grand intérêt par son caractère panoramique, et par l'importance que Laplace - avec son époque - attribue aux applications des connaissances scientifiques. Tout ce à quoi le calcul des probabilités a été appliqué y est passé en revue : jeux de hasard, démographie, théorie des assurances et des rentes viagères, loi et correction des erreurs d'observation ; en passant par des considérations sur la validité des témoignages, la fonction et la composition des assemblées délibérantes et les jugements des tribunaux, dans lesquelles le lecteur contemporain décèlerá sans peine de la naïveté idéologique, au risque peut-être de méconnaître qu'il y avait là, aussi, un premier essai d'approche scientifique de problématiques qui restent vivantes : la théorie des Jeux, après tout, procède elle aussi de ces mêmes préoccupations.

Les passages les plus intéressants de cette revue de détail concernent la démographie et la théorie des erreurs d'expérience, deux domaines où Laplace fait figure de pionnier. À propos de ce qu'il appelle les « applications à la philosophie naturelle $"$, il faut remarquer qu'elles ne concernent qu'indirectement la connaissance des phénomènes naturels. Leur objet d'application directe, c'est la méthode scientifique, qui ressortit en réalité au domaine des sciences de la société. On ne remarque pas toujours que le calcul des probabilités a été la première en date de celles- 
ci, et n'a été que cela pendant près de deux siècles après son coup d'envoi par Pascal et Fermat. C'est seulement avec Maxwell, Boltzmann et Gibbs qu'il a fait son entrée par la grande porte dans les sciences de la nature. Laplace aurait sans aucun doute salué cette entrée avec enthousiasme ; il ne pouvait l'anticiper.

En ce qui concerne la démographie, Laplace n'a pas seulement fait œuvre de théoricien, en commençant à déceler les pièges du calcul démographique ; il a eu une conscience aiguë de l'importance de ce que nous appellerions aujourd'hui les bases de données et fortement contribué à leur développement, y compris par son influence politique. Son souci de disposer de données de fait, bien formées et abondantes, s'est d'ailleurs étendu aux applications des sciences de la nature (la théorie des marées a été une de celles qui lui ont le plus tenu à cœur).

Laplace, comme rationaliste convaincu et comme chercheur, ne pouvait manquer de s'interroger sur la rationalité de la découverte scientifique. Faisant peu de part à l'intuition du chercheur, il recense les moyens de découverte qui sont d'abord, classiquement, "l'induction et l'analogie ", qu'il illustre par des exemples. Il raisonne en mathématicien. L' « analogie », pour lui, c'est avant tout ce qu'on appellerait aujourd'hui le prolongement ou l'extension d'un concept (cf. les concepts d'exposant fractionnaire de Wallis, puis d' " exposant variable » de Leibniz, p. 70). L' " induction " est d'abord conçue sur le modèle du raisonnement par récurrence : on soupçonne une règle, puis on la démontre. Laplace ne se satisfait pas aisément de preuves expérimentales ; la certitude n'est pas le fruit d'une régularité expérimentale de plus en plus convaincante, mais d'une explication théorique. Après avoir énuméré plusieurs phénomènes dont il avait lui-même trouvé une explication théorique délicate, il conclut : « dans les cas que je viens de considérer, la solution analytique des questions a converti la probabilité des causes en certitude » (p. 102).

Mais il n'y a pas que l'analogie et l'induction. En passant, sans insister, il fait une remarque beaucoup moins classique sur l'ambiguïté des formulations mathématiques comme source de découverte, qui fait penser à certaines thèses de $\mathbf{M}$. Loi sur la fécondité heuristique de l'ambiguïté : notant que les fonctions génératrices sont une représentation équivalente des équations aux différences finies, il remarque que « c'est dans de semblables transformations que réside le pouvoir de l'Analyse ".

On trouvera enfin dans l'Essai, au détour d'un paragraphe, de façon disséminée, des aperçus prospectifs qui frappent par leur modernité, tant à propos de problématiques scientifiques que d'applications des connaissances à des problèmes pratiques. Quelque chose a décidément changé dans les dernières décennies du Xvi!le siècle. Nous avons déjà vu que par la théorie des erreurs d'observation et par la démographie, Laplace entrait de plain-pied dans le domaine de la statistique ; noté ce qu'avait de nouveau son souci de développer des bases de données ; remarqué au passage ce qu'il y avait de moderne dans ses tentatives de réflexion scientifique sur les structures de la société, malgré la tare d'une contamination idéologique dont notre époque ne tolérerait plus qu'elle fût aussi naïvement évidente, sans pour autant en être elle-même exempte. Mais au passage, on peut relever d'autres détails : ici, une ébauche de raisonnement typiquement ergodique (à propos de l'hypothèse de la « nébuleuse primitive », dont il croit 
retrouver l'évolution historique dans la collection des états actuels des nébuleuses observées dans le ciel, p. 111) ; là, avant Malthus (et Meadows !), une référence à la régulation des populations par le niveau des subsistances (p. 147); etc. Des idées prospectives de Laplace, c'est probablement le projet d'une psychologie scientifique (repris de $\mathrm{Ch}$. Bonnet) qui aura le plus vieilli.

En revanche, il est à peine besoin d'insister (cela est suffisamment connu) sur ce qu'avaient de révolutionnaire les idées de Laplace sur l'histoire de l'univers - même si Kant l'avait précédé dans cette voie, et même si sa pensée n'allait pas sans contradictions : c'est ce même Laplace, qui développait l'hypothèse de la nébuleuse primitive, qui pouvait écrire avec semble-t-il une égale conviction, et sans formuler de réserve, que « nous sommes portés à croire que l'ordre, suivant lequel nous voyons les choses se renouveler sur la terre, a existé de tout temps et subsistera toujours ".

Laplace, dira-t-on, était un " scientiste ". Sans nul doute, il croyait avec son siècle, et à un degré extrême, aux pouvoirs de la science. Il les croyait illimités dans leur principe. Mais il savait aussi leurs limites dans la réalité concrète. D'autre part, on peut inviter le lecteur du $\mathrm{XX}^{\mathrm{e}}$ siècle à se poser la question suivante : quelle était au début du XIXe siècle la croyance la plus féconde : celle qui prédisposait à croire au pouvoir de la science de pénétrer dans la connaissance des êtres vivants, en tant qu'individus et dans leur vie sociale, ou celle qui posait des barrières de principe à ce pouvoir ? Il faut aussi donner acte à Laplace de ce que la pointe de son " scientisme " est vigoureusement dirigée, non seulement contre les illusions du sens commun, mais aussi contre tous les charlatanismes. L'Essai contient aussi des pages vigoureuses sur ce thème, qui n'ont rien perdu de leur actualité.

Dans la préface qu'il a rédigée pour cette publication, $R$. Thom fait un sort tout particulier au déterminisme de Laplace, dont il esquisse la critique du point de vue des mathématiques contemporaines. Les pages qu'il écrit à ce propos sont à verser au dossier de ce débat, qui se poursuit. Elles mériteraient sans nul doute une discussion spéciale, qui ne saurait être brève. Peut-être aussi serait-ce exagérer l'importance du déterminisme dans la pensée de Laplace que de le privilégier à ce point dans le cadre du présent commentaire. Ce que nous aurions à dire de cette préface concernerait bien plus la pensée de Thom que celle de Laplace. Ce ne serait sans doute pas moins intéressant - mais c'est une autre histoire.

Jacques BONITZER.

Théodore F. GERAETS, Lo Spirito assoluto come apertura del sistema hegeliano. Naples, Bibliopolis, 1985. 14,5 × 21,2, 98 p. ( $($ Memorie dell'istituto italiano per gli studi filosofici $", 9)$.

T. F. Geraets cherche à répondre, dans cette reprise de trois conférences données à Naples en 1982, à la question de savoir si la théorie de l'Esprit absolu qui, de toute évidence, se donne comme la conclusion du système hégélien, opère une clôture dogmatique ou une ouverture critique de la pensée spéculative. C'est vers 
une auto-compréhension ouverte du système - à l'instar de B. Bourgeois, G. Jarczyk, D. Rosenfield - que s'oriente la réponse de l'auteur. L'originalité de son interprétation réside dans l'exploitation du concept de " reconnaissance " étendu de son contexte phénoménologique initial aux trois sphères de la philosophie de l'histoire, de la philosophie de la religion, de "la philosophie hégélienne de la philosophie " (p. 70), modalisant diversement l'ouverture à l'infini de l'esprit absolu. L'être-reconnu de l'infini par l'esprit fini de même que la reconnaissance de l'esprit infini par l'esprit fini, « la réalisation d'un tel processus de reconnaissance est sans doute une tâche qui ne sera jamais définitivement achevée " (p. 38). " L'Esprit absolu comme Esprit du monde ", d'abord (p. 11-38) : notre temps, le temps du monde de l'interdépendance Est-Ouest des idéologies, de l'interaction Nord-Sud des économies, doit-il se comprendre à partir de la thèse d'un État mondial en cours d'achèvement politique (résidu d'optimisme organiciste-romantique de Hegel) ou de la thèse d'un dialogue ouvert par les grandes individualités pensantes - distinctes des héros politiques " rusés » de la raison - du type de celles de Socrate ou des penseurs " critiques " ? L'auteur envisage la seconde réponse : penseurs, intellectuels et « conseillers » doivent avoir leur rôle à jouer dans le dialogue d'intégration à l'infini de principes politiques hétérogènes. Le Hegel « vivant " serait, pour notre temps, celui de la dialectique du mal (violence extrême) et de son pardon (compréhensif), plus que celui du héros politique homogénéisant violemment les peuples, les « modelant ». T. F. Geraets joue « le mal et son pardon » contre « la ruse de la raison », pour ouvrir l'esprit absolu dans le champ de l'histoire politique.

Seconde ouverture possible de l'Esprit absolu : celle de la religion (p. 39-64). $\mathrm{Si}$, dans la première partie de l'ouvrage, il était refusé à l'État mondial d'unifier « absolument » les esprits, c'est, dans la seconde partie, à travers un dialogue des communautés religieuses qu'est pensée l'ouverture à et de, l'Esprit absolu : " la communauté des croyants se révèle, pour Hegel, comme l'être même de l'Esprit absolu » (p. 45).

Ainsi, la religion absolue, « au moins pour nous qui tentons de prolonger la pensée hégélienne ", est l'expression d'une réconciliation, certes, éternelle en soi, mais qui ne demeure vivante qu'à travers un dialogue des communautés reflétant toutes quelque chose de Dieu, s'interrogeant elles-mêmes, c'est-à-dire s'interrogeant entre elles (cf. p. 55). Dès lors, il deviendrait essentiel de comprendre que ce n'est pas tant le progrès infini que critiquerait Hegel (p. 58) que son interprétation dualiste d'entendement sur le mode d'une conformité extérieure, à l'infini, d'une réalité finie à un modèle unique et transcendant. « Le vrai ressort du progrès à l'infini " (p. 59) est la présence réciproque de l'un dans l'autre, sur le mode d'un dépassement de soi qui, pour l'infini, est aliénation éternelle de soi et, pour le fini, reconnaissance sans fin de son autre dans le temps.

La troisième ouverture du système hégélien est représentée par la construction de l'Idée de la philosophie dans l'Encyclopédie (p. 95-98). L'auteur y reprend son article publié en 1975 dans les Hegel-Studien, B. 10, p. 231-254. Le premier syllogisme (Enc. § 575) expose la Logique comme éternelle pensée de la pensée, " dans un régime de nécessité qui semble "naturelle", extérieure au sujet philosophant » (p. 80) : Logique, Nature, Esprit. Ce dernier recueille la science, l'ab- 
strait de la nature et de lui-même, sans la produire. Et il est d'abord vrai que la Logique est ce qui « anime " en soi l'Esprit. Mais une seconde lecture du système est nécessaire : Nature, Esprit, Logique ( $\$ 576$ ). Le Savoir logique est alors le produit d'une auto-réflexion de l'Esprit qui, dépassant la nature dans son histoire, est produite par un esprit singulier pensant la totalité. Pour exprimer simplement ces deux « lectures » du savoir absolu, nous dirons que ce dernier est tout à la fois ce que l'Esprit fini " trouve " au fond de lui-même et dans la nature, comme ce qui s'impose à lui, mais, simultanément, " écrit " et produit activement à travers le langage philosophique d'une époque et d'un temps susceptibles d'être eux-mêmes dépassés, c'est-à-dire à la fois repris et prolongés à l'infini.

Si la première lecture révèle une totalité de pensée achevée et en ce sens « fermée sur elle-même " (p. 83), la seconde, faite du point de vue du sujet philosophant lui-même, doit sans doute nécessairement demeurer " une pensée ouverte " (ibid.), un effort de totalisation en cours. Le troisième syllogisme tâche de penser l'unité de ces deux lectures (\$ 577) : la Science est à la fois l'Idée éternelle " visée " par l'Esprit et ce dont son effort même procède, puisqu'il se pense comme le recueil du logos naturellement aliéné : Esprit-Logique-Nature. Le troisième syllogisme « reconnaîtrait » que le sujet fini ne peut évidemment cö̈ncider, définitivement, avec le savoir absolu éternel « apparent » du premier. Ainsi que nous le faisions déjà remarquer à propos d'un livre de D. Rosenfield ' ${ }^{1}$, il nous semble difficile de justifier en droit cette ouverture du système du savoir philosophique sans reprendre le concept kantien d'Idée régulatrice afin de penser l'écart entre l'Idée d'une systématicité close (premier syllogisme) et la systématisation historiquement produite par " un sujet philosophant " (T. F. Geraets) qui se comprend comme le retour à soi du concept dans le troisième syllogisme où il se pense tout à la fois comme le mouvement de l'Être et l'activité essentielle du connaître qu'est l'Esprit absolu.

André STANGUENNEC.

Georges KalinowSKI, Sémiotique et philosophie : à partir et à l'encontre de Husserl et de Carnap. Paris-Amsterdam/Malakoff, Distique/Hadès : Benjamins, 1985 . $14 \times 21,290$ p. ("Actes sémiotiques »).

La thèse centrale de ce livre est qu'il n'y a pas de sémiotique sans philosophie - ou plus exactement sans ontologie - et que toute philosophie se doit d'avoir une sémiotique. Mais, contrairement à la tradition récente de la philosophie " analytique ", l'Auteur ne soutient pas que la sémiotique ou la philosophie du langage est la philosophie première et que l'ontologie vient après (comme le soutient par exemple M. Dummett). G. Kalinowski demeure traditionnel sur ce point, et il mesure les conceptions sémiotiques à l'aune de leur ontologie, et non pas inversement.

1. Revue de synthèse, I11' S., 117, janv.-mars 1985, p. 115. 
L'ouvrage est divisé en deux parties. Dans la première, l'Auteur passe en revue diverses théories de la signification du passé, pour en dégager les lignes de force et de tension, et surtout pour les critiquer. Selon lui, les « bonnes » conceptions sémiotiques sont celles qui, à l'instar de celle d'Aristote dans le Peri Herméneias, dégagent deux dimensions de la signification : la désignation et la signification. Les Stoïciens et saint Augustin, puis Thomas d'Aquin, relèvent pour G. Kalinowski de la même conception équilibrée des signes. Les conceptions plus modernes, qu'il expose, celles de Frege, de Husserl et de Carnap, témoignent toutes, à ses yeux, d'un déséquilibre entre signification et désignation. Husserl privilégie la première, Carnap la seconde. G. Kalinowski reconnaît que ce n'est pas totalement vrai de Frege ni de sa distinction entre le Sinn et la Bedeutung d'une expression. Mais il le taxe néanmoins d'incohérence, parce qu'oscillant entre réalisme ontologique et idéalisme platonicien (les Gedanken de Frege ne sont pas de ce monde). Husserl et Carnap sont analysés plus abondamment et ont droit chacun à un chapitre. G. Kalinowski, conformément à sa thèse principale, critique la sémiologie de Husserl à la fois parce que, à ses yeux, et sémiotiquement et ontologiquement infondée. Ce qu'il reproche à Husserl est d'être un idéaliste qui ne croit pas que le monde extérieur existe. L'ego transcendantal n'est que le masque de la schizophrénie du phénoménologue (p. 57, Marc Oraison à l'appui), Quant à Carnap, il est au fond nominaliste, malgré son refus des « questions externes » par rapport à la sémantique (p. 126). Cette première partie est sans doute la moins convaincante du livre. On peut s'accorder avec l'Auteur sur la maxime, « telle philosophie, telle sémiotique ", mais cela l'autorise-t-il à critiquer sur des bases ontologiques uniquement une sémiotique donnée ? De ce point de vue, il avoue très explicitement sa préférence pour l'ontologie aristotélicienne et la sémiotique qu'il trouve dans cette tradition. Selon lui, une bonne sémiotique doit dépendre d'une " ontologie réaliste " pour laquelle : 1) le monde extérieur existe ; 2) les objets intentionnels ne sont pas des êtres réels ; 3) notre pensée, cognitive ou non, comporte des vécus psychiques dont les contenus, pris in abstracto, sont appelés " concepts au sens logique »;4) ces concepts sont immatériels comme la pensée qui les produit et les supporte existentiellement (p. 161). C'est son droit, mais on a trop souvent l'impression que le seul argument avancé contre les thèses examinées est qu'elles n'entrent pas dans ce cadre réaliste. Trop d'analyses sont (de l'aveu même de l'Auteur) sommaires. Et surtout, on ne voit pas pourquoi certaines grandes conceptions du passé sont passées sous silence : rien sur Leibniz (sauf plus loin sur les « mondes possibles »), Bolzano, ni sur les médiévaux. Rien sur Peirce, peut-être le plus grand sémioticien de l'histoire de la philosophie (dont l'Auteur ne goûterait sans doute pas le réalisme scotiste quant aux universaux).

On aura compris que le but de cette première partie est seulement de préparer, polémiquement, les thèmes de la seconde, où G. Kalinowski développe ses propres idées sémiotiques. Ici, le logicien des normes et des modalités qu'il est a beaucoup plus à nous apprendre. Il consacre trois chapitres originaux respectivement à la distinction entre désignation et signification, à celle entre vérité analytique et synthétique, enfin à la conception de la vérité contenue dans les sémantiques « des mondes possibles ». Les présupposés demeurent, comme on peut s'y attendre, aristotéliciens et thomistes. Il y a des analyses intéressantes sur la distinction 
entre êtres réels et objets intentionnels, entre analyticité $a$ priori et $a$ posteriori (l'Auteur pensant avec Thomas d'Aquin que la connaissance est abstractive et que les signifiés le sont aussi, il ne peut $\mathrm{y}$ avoir pour lui de pures vérités $a$ priori, ou plutôt si elles existent, elles sont vides). G. Kalinowski dénonce une conception implicitement nominaliste dans les théories sémantiques de Montague-Lewis, ou bien faussement réaliste : le possible n'est pas une forme d'être, mais n'est qu'une potentialité. Ici encore, ses analyses ne convainquent que pour autant que l'on admette ses prémisses ontologiques. Mais il montre avec talent que l'on n'est pas obligé d'accepter les conceptions de la vérité, des modalités, et de la sémantique qui prédominent chez les fondateurs de la sémantique modale moderne. La place manque pour discuter toutes ses vues dans cette seconde partie. Le grand mérite du livre est de montrer qu'il y a, à côté de certaines conceptions dominantes de la sémantique et de la théorie de la signification dans le monde anglo-saxon, place pour une sémiotique qui est beaucoup plus proche d'une ontologie formelle, dans la tradition aristotélicienne-brentanienne-polonaise-mitteleuropéenne (pour la qualifier largement) que des ontologies nominalistes héritées du positivisme. On peut regretter que G. Kalinowski n'ait pas consacré encore plus de place à son argument en faveur de la sémantique " réaliste " bien comprise et moins à la polémique. Il manque aussi un argument en faveur de la thèse principale du livre : la dépendance de la sémantique par rapport à l'ontologie, ou tout au moins une considération plus attentive de la thèse selon laquelle le réalisme pourrait être une thèse sémantique.

Pascal ENGEL.

Manifeste du Cercle de Vienne et autres écrits. Carnap, Hahn, Neurath, Schlick, Waismann, Wittgenstein. Sous la dir. d'Antonia Soulez, textes trad. de l'allemand par B. Cassin, C. Chauviré, A. Guitard, J. Sebestik, A. Soulez, J. VICKers. Paris, P.U.F., 1985. 13,5 × 21,5, 364 p., lexique, glossaire, bibliogr., index ("Philosophie d'aujourd'hui »).

L'ouvrage met à la disposition des lecteurs français, pour la première fois, le texte de la « brochure jaune » par laquelle le Cercle de Vienne avait pris position avec fracas sur la scène philosophique en 1929. Les raisons qui font du Manifeste une déclaration d'indépendance à l'égard de la philosophie traditionnelle sont analysées par A. Soulez dans son introduction ; J. Sebestik place ensuite les textes de Neurath, Hahn et Carnap dans la perspective du développement de la philosophie autrichienne, et $C$. Chauviré analyse le rapport complexe de Peirce, et plus généralement du pragmatisme américain, avec les thèses du Cercle. Cinq autres textes caractéristiques des débuts du groupe sont également traduits : « Le dépassement de la métaphysique par l'analyse logique du langage " de Carnap (1931 et 1957), "Vécu, connaissance, métaphysique » de Schlick (1926), « Entités superflues » de Hahn (1929), " Énoncés protocolaires » de Neurath (1932), et «Quelques entretiens de Wittgenstein » (notes prises par Waismann entre 1929 et 1932). Chaque texte est précédé d'une note historique, et annoté. L'addition 
d'un lexique de la traduction, d'un glossaire, d'une bibliographie importante et de deux index font du livre une sorte de Source Book du Cercle de Vienne dans les années de son émergence. Il faut, à tous égards, saluer cette publication 1 .

L'objet explicite des auteurs est de recomposer l'image que le Cercle a voulu donner de lui-même à l'époque du Manifeste de 1929 en lui rendant à la fois sa violence et son contexte. Pour la violence, on sait que peu de mouvements philosophiques ont été aussi explicitement formés pour et par la dénonciation des erreurs précédentes. La lecture de ces premiers textes le montre bien : on y chercherait en vain le sens des questions aporétiques, fût-ce en vue de mettre en difficulté la tradition philosophique. L' " analyse logique " y est utilisée comme argument d'autorité, le " dépassement de la métaphysique " y est mis au service d'une véritable préparation d'artillerie, et l'ironie à l'égard des effets, pesants il est vrai, de l'idéalisme allemand sur la conscience européenne ne diminue en rien l'indéniable esprit de sérieux de la formulation du programme pour toute philosophie future que propose le Manifeste. Le ton de ces textes des origines est-il la source lointaine de l'intensité irrationnelle de certains débats actuels sur l'empirisme logique versus la " philosophie continentale " ? Les auteurs ont pris le seul parti possible : loin d' " adoucir les traits de l'empirisme logique viennois ", ils suggèrent d'abord que cette violence théorique doit être rapportée à une " situation " en un sens presque sartrien, à un entrelacement de raisons sociales, politiques et institutionnelles perçues avec une netteté particulière par Neurath. La hiérarchie allemande des savoirs reproduisait dans l'enseignement universitaire la prééminence des sciences de l'esprit au détriment des sciences de la nature, ce qui affublait la métaphysique d'un cortège de pensées très impures ; l'essor d'idées démocratiques de rénovation sociale et scolaire semblait donner un fondement à la tentative de promouvoir un rationalisme universalisant libéré de la tradition spéculative, d'ailleurs radicalement mise à mal par le développement de la physique (« désagrégation de l'a priori », etc.). En d'autres termes, la violence philosophique, le concept même de démarcation, étaient les effets d'un contexte de pouvoir, et le Cercle de Vienne était voué à devenir ensuite, en dehors du sol autrichien, un positivisme irrémédiablement « déraciné ». Il est probable, en effet, que la difficile insertion de l'empirisme logique dans la philosophie américaine (malgré les apparences) a été due au caractère tout différent de la tradition en place : celle de Dewey, notamment. Quoi qu'il en soit, les auteurs montrent très bien la brièveté et la complexité de la durée de vie autrichienne du groupe : né dans un rapport équivoque à Schlick et à Wittgenstein, le Cercle se présente en 1929 comme la première percée des idées positivistes (sans mentionner le « manifeste » de 1911 signé par Mach, Petzoldt, Hilbert, Klein, Helm, Freud et Einstein) ; il se constitue en 1924 sur la proposition de Feigl et de Waismann, bénéficie de l'arrivée de Carnap à Vienne en 1926, annonce sa révolution au Congrès de Prague en 1929, et se dissout dans sa propre internationalisation dès 1930.

1. Signalons également la parution des Actes des Journées sur le Cercle de Vienne, organisées en 1983 par A. Soulez et J. Sebestik : Le Cercle de Vienne. Doctrines et Controverses, Paris, Klincksieck, 1986. 
Officiellement, le mouvement sera supprimé par les autorités en 1938, comme " foyer suspect de judaïser la science".

Cette brièveté et le fait que le groupe n'était d'abord cimenté que par son opposition à la philosophie universitaire et son horreur très générale de la tradition ont eu assez naturellement des conséquences sur l'unité des thèses revendiquée dans le Manifeste. Avec soulagement, on observe que, bons philosophes, les membres du Cercle n'étaient d'accord sur à peu près rien. Ni sur les « énoncés protocolaires ", c'est-à-dire sur le problème d'un fondement de la connaissance, à propos desquels Neurath reproche à Carnap de rester encore prisonnier de la manie traditionnelle de la fondation. $\mathrm{Ni}$, par extension, sur la nature d'un « langage primitif ». Ni sur les moyens de résoudre la contradiction entre le projet d'une langue universelle ralliant le consensus et la thèse essentielle que toute philosophie n'est que le développement des possibilités linguistiques offertes (la métaphysique occidentale étant ainsi « le résultat d'opérations construites sur la copule "être" ", une idée d'ailleurs répandue à l'époque). Ni, enfin, sur la meilleure définition du " vérificationnisme ». Curieusement, tous ces problèmes qui ont investi depuis l'épistémologie contemporaine sont traités d'abord à l'emporte-pièce, par assertions, critiques, corrections, " libéralisations " : de la philosophie « en acte ", selon l'expression de Schlick. Ce n'est certes pas cette accumulation de thèses que l'on reprochera au Cercle, sauf à suggérer, là encore, qu'un grand nombre d'obscurités inutiles en ont, en fait, encombré l'héritage après 1940. En revanche, il est nécessaire de confronter les écrits du groupe à leur finalité explicite : le dépassement de la métaphysique y est-il opéré ? La nouvelle philosophie proposée est-elle en accord avec le « fait » des nouveaux développements de la mathémathique et de la physique?

Sur le premier point, la critique systématique du texte de Heidegger « Was ist Metaphysik ? " par Carnap était à l'évidence un exemple privilégié. Heidegger survécut facilement à sa propre " déconstruction », et $\mathbf{A}$. Soulez rappelle à juste titre dans son introduction qu'en marquant l'achèvement du mode de pensée ontologique (dans sa relation à la possibilité des sciences positives), il mettait bien, lui aussi, en question la paranoïa du "vouloir fonder ". Mais la confrontation eut l'avantage de permettre la discussion d'une question délicate : comment " dépasser la métaphysique " sans être toujours déjà prisonnier de son langage ? Critiquer la tradition spéculative au moyen d'un langage qui ne lui appartient pas, comme le recommande Carnap, cela autorise-t-il une clarification suffisante des énoncés métaphysiques ? Si oui, il faut en conclure que le système des énoncés est séparable des formes linguistiques - thèse précisément contradictoire avec l'assertion précédente que la métaphysique n'était que le développement de formes linguistiques particulières. Par suite, du point de vue de Carnap (en 1929), seule l' " exclusion " pure et simple de la métaphysique devrait être efficace : " l'apparence arbitraire de l'appel à la méthode logique vient de ce qu'il faut intervenir extérieurement à la métaphysique pour résoudre l'aporie de ce dépassement " (p. 76). Mais, du même coup, c'est l'argument d'autorité qui devient indépassable. Il est frappant qu'exactement en même temps, à propos non plus de l'ontologie moderne mais de la physique classique, les physiciens du groupe de Copenhague aient rencontré dans toute son ampleur une question similaire : 
comment dire quelque chose sur les atomes tout en opérant une critique radicale du discours antérieur de la physique ? Et une alternative identique : renoncer entièrement au genre de ce discours (et ne plus parler que dans le formalisme, sans référence à des états de choses " réels »), ou bien rester à l'intérieur de la langue de la physique classique tout en sapant à la base sa prétention à être une description vraie. Que le Cercle de Vienne, pour sa part, ait voulu dépasser la métaphysique en en détruisant le vocabulaire, est une chose ; qu'il y soit parvenu est beaucoup plus douteux, dans la mesure où, du moins à cette époque, il se trouve acculé à des procédures d'exclusion assez tâtonnantes. Entre la lourdeur de l'idéalisme allemand enseigné dans les universités autrichiennes, et la complexité de la question de l'ontologie moderne telle qu'elle apparaissait par exemple tant à Heidegger qu'à Heisenberg, il y eut peut-être une confusion de cibles.

Quant au second point, laissé de côté par les auteurs pour des raisons d'économie générale de l'ouvrage, il engage de toute façon tant de problèmes différents que l'on comprend qu'il soit rarement traité. On maintiendra tout de même qu'il paraît important de juger sur pièces un mouvement philosophique qui se présente comme une rupture avec toute la philosophie classique au nom de la promotion d'une théorie de la science, fondée sur les récents progrès des « sciences inductives ". Quel " usage " le Cercle fit-il de ses multiples évocations d'Einstein, de Hilbert ou de Heisenberg ? En se réclamant, notamment, de la théorie de la relativité et de la mécanique quantique, en quoi a-t-il apporté des éclaircissements majeurs sur leur structure conceptuelle - comparables, par exemple, à ceux donnés par Pauli —, ou sur la nature des théories physiques - comparables à ceux donnés par Heisenberg -, ou sur des points plus détaillés comme la définition des observables (ici, le malentendu est complet entre l'usage du mot en physique et son usage dans Erkenntnis), ou les concepts de substance, d'individualité, d'identité ? Si les réticences de Schlick, et surtout de Wittgenstein sont clairement mises en évidence, il faudrait aussi mentionner les critiques très vives des physiciens eux-mêmes et de philosophes comme Albert Lautman (sur l'interprétation de la mathématique hilbertienne, en particulier). Enfin, pour en revenir à Heidegger, une question devrait être posée et développée : une théorie de la science peut-elle être une " conception du monde " ? Le projet du Cercle de 1929 ne tombe-t-il pas sous le coup de la critique de la notion de «Weltanschauung 》 donnée par Heidegger, par exemple dans les Problèmes fondamentaux de la Phénoménologie ? Il est vrai que Carnap abandonne le mot en 1931. Mais on observera que cet abandon est justifié par des raisons identiques à celles de Heidegger : il ne faut pas confondre la « théorie » (resp. la « philosophie ») avec l'expression d'un sentiment de la vie.

Toutes ces interrogations tourneraient aisément en faux procès, si l'on ne remarquait pas qu'en réalité l' "épistémologie » du Cercle ne se présente nullement comme une explication des contenus ou des problèmes des mathémathiques ou de la physique. Il s'agit plutôt de dénoncer soit le désintérêt de la tradition spéculative pour les sciences en général et la révolution qu'elles introduisent en philosophie, soit, pire, le réductionnisme que la branche néo-kantienne tente d'opérer. Le paradoxe est ainsi que la philosophie scientifique du Cercle, pour être une machine de guerre contre les obscurités de la pensée, ne se transforme pas 
pour autant en philosophie des sciences, et même manque un aspect essentiel de la physique quantique : la disparition des intuitions et l'aveuglement corrélatif des concepts.

Ajouter les entretiens de Wittgenstein avec Waismann était une excellente idée : sa proximité même, la densité de ses remarques, mettent dans une souriante perspective les enthousiasmes de la brochure jaune.

Catherine Chevalley.

Jean Largeault, Principes de philosophie réaliste. Paris, Klincksieck, 1985. $16 \times 23,272$ p. (« Philosophia », 10).

Le savant épistémologue de Créteil (Université de Paris-XII) offre ici, comme à son habitude, un livre riche d'aperçus, caractérisés par la sûreté de l'information et la fermeté du jugement : un livre à la hauteur de son sujet, ce que par ailleurs il n'est pas fréquent d'avoir à reconnaître dans les ouvrages d'épistémologie générale, trop souvent cantonnés, en effet, dans l'exposé passe-partout, vieillot et rhétorique. Comme on l'admet unanimement aujourd'hui si l'on veut travailler sérieusement : " on ne peut pas discuter de l'explication scientifique sans se reporter à l'histoire des sciences " (p. 7). L'auteur a conscience que l'épistémologie est devenue « inintéressante " (p. 9), " stérile » (ibid.), malade du " sentiment de la monotonie " (p. 10, ici une critique due à son maître René Poirier), sentiment évidemment fort opposé à ceux que peut susciter la variété de l'histoire. À l'encontre des diverses ontologies que rencontre l'historien du savoir scientifique, le formalisme positiviste se refuse aux hypothèses sur la nature des objets, dans une longue tradition qui remonte, dit l'auteur, au XVIII' ${ }^{e}$ siècle (cf. p. 7), mais dont on trouve moins systématiquement les racines et déjà les insuffisances au siècle précédent (cf. l'attitude de Galilée à l'égard des difficultés des fontainiers). Les positivistes connaissent la « générativité » (p. 8, cf. p. 49), au sens de la linguistique générative de Chomsky, mais la coupent de toute ontologie et la réduisent à une démarche logico-déductive. L'auteur prend nettement parti pour un " réalisme " au sens où ce terme s'oppose à un " idéalisme " en refusant que le sujet connaissant soit fondateur : « les choses ne sont pas parce que je les pense " (p. 12), ni parce que nous les pensons, ni non plus parce que l'homo sapiens les pense. On remarquera que Largeault n'exclut pas du réalisme, terme qui prête à toutes les équivoques quand il n'est pas spécifié comme ici, une philosophie comme celle de Gilson (cf. p. 15, p. 16, n. 8 et n. 16) où les choses dépendent d'une pensée supérieure à celle du je singulier, collectif ou universel. Il prend donc " réalisme " dans une acception non absolument discriminante et il ne refuse pas toute forme d'idéalisme ou de spiritualisme, mais seulement des formes d'idéalisme qui conduisent au transcendantalisme kantien ou au fantôme du " solipsisme " (p. 9). Le mot d'ordre provocant de son livre pourrait bien être : " $\mathrm{Ne}$ se débarrassera-t-on jamais de Kant ? " (p. 63, n. 15). Son horizon reste donc en deçà de la question fondamentale ou primordiale qui n'est pas de savoir si nous sommes ou non « le fondement de l'ordre que nous découvrons dans la 
nature " (p. 271), mais bien plutôt si cet ordre se subordonne un désordre dérivé ou si c'est au contraire le désordre qui est à l'origine de l'ordre ${ }^{1}$.

Le chapitre premier traite de l'explication. L'intérêt se porte ici sur « une pluralité de types » (p. 18) et de niveaux, ainsi que sur les passages de la description à la théorie : « au lieu de s'obstiner à séparer, décrire et expliquer, ce qui ne se peut qu'idéalement, il vaudrait mieux considérer les deux activités comme jointes. La différence est de niveau » (p. 21). Si un formalisme d'esprit subjectiviste n'a au mieux qu'une valeur provisoire et n'explique rien, il apparaît au contraire que, dans une optique réaliste, « les considérations de forme ont été depuis 1905 à l'origine de tous les progrès de la physique ". On comprend mieux la gravité depuis qu'on l'a géométrisée (p. 55 et cf. p. 52 : « l'explication par les forces n'est pas essentielle au mécanisme ", ajoutons que cela se voit bien chez ses deux grands pionniers, Descartes et Hobbes) ${ }^{2}$. Modestie et bon sens en même temps : " les schémas sur quoi repose l'explication sont aussi bien ceux du sens commun " (p. 58), pas d'explications ultimes ou complètes (cf. p. 22), mais un effort pour réduire " l'arbitraire de la description » (Thom, cité p. 36, repris p. 58, référence p. 112).

Le court chapitre II sur l'Induction (thème déjà amorcé p. 8 sqq.) nous la montre reposant sur « la stabilité des espèces naturelles " (p.11) évidemment irréductible à quelque logicisme ; toute induction, ancienne ou moderne (classes ou relations) comporte un risque et est susceptible de révision. Le chapitre III a pour titre : « De l'analyse des causes. » La causalité, était-il déjà dit plus haut (p. 52), n'est pas à mettre au rang des "vieilles lunes », elle ne se laisse pas résorber dans la régularité légale, non plus que le hasard dans une détermination définie. " Le hasard se conçoit toujours comme ce qui déborde un système d'information donné " (p. 101), il y a analogie profonde entre hasard et vérité. L'auteur me paraît sous-estimer la théorie de Cournot qui, peut-être, va loin avec la notion de multiplicité incoordonnée qui rendrait compte très bien de l'impossibilité d'unifier un hasard sans contradiction (" toute théorisation est déterministe " ${ }^{3}$, p. 109). L'orientation réaliste au sens de Gilson va de pair comme il est naturel avec le souci de reconnaître, de manière aristotélicienne, une variété de types de causes : « préciser, en chaque circonstance, quel concept de cause nous employons, est à notre portée. L'intéressant serait de savoir avec quelle sorte de cause (forme ou force) opère la nature » (p. 136).

Le chapitre IV (" La forme et le mouvement ») développe des indications du chapitre premier sur l'explication en se concentrant sur la théorie des catastrophes de M. René Thom, dont l'auteur se reconnaît aujourd'hui le disciple. Le chapitre V, par opposition, achève l'exécution de "l'épistémologie néopositiviste ", notamment de Quine et de Popper (plus goûté naguère, répudié aujourd'hui avec énergie, on n'est plus au temps de l'Enquête sur le Nomina-

1. Tendance spiritualiste ou idéaliste au sens majeur : cf. René Tном, « Halte au hasard, silence au bruit ", Le Débat, juil.-août 1980 - contre la tendance matérialiste de Prigogine.

2. Il est par ailleurs difficile de digérer l'affirmation suivante (p. 50) : « Descartes croyait l'expérience superflue. " Atténuation, p. 156.

3. C'est-à-dire réduit en système unifié les déterminations. 
lisme). L'irrationalisme de Popper (p. 188) ne tient pas seulement à son logicisme et à son « déductivisme " (p. 186), il tiendrait plus foncièrement, selon l'auteur, à son refus d' " un discours unitaire » (p. 187) qui serait caractéristique de la science et pas seulement d'une pensée éléatique. On retrouve ici l'opposition de l'idéalisme au sens majeur (comprenant aussi bien le thomisme que - calembour p. 159 - la philosophie de Thom, c'est-à-dire les " réalismes " de l'identité fondamentale) et du matérialisme au sens majeur, très mal défini à mon sens et disqualifié d'avance comme " réductionniste " (p. 171), d'où l'absurdité de l'exercice de la p. 173, associant contradictoirement le primat de l'unité et celui de la diversité. L'option matérialiste serait du reste fort mal représentée par Popper, à qui Largeault accorde bien vite le beau titre de " philosophe dissonant " (p. 200) pour l'en accabler. En revanche, la théorie des catastrophes est représentative d'un élan vers l'unité, par intégration des discontinuités dans des fonctions continues (cf. p. 148), ce qui ne veut pas dire que l'idéalisme triomphe à travers elle, du seul fait que l'on a tendance à l'en extrapoler ; on en dirait autant en cosmogonie de la "grande théorie unifiée " qui unifie grandement le réel, mais non pas totalement.

« L'idée de philosophie de la nature » (chapitre de conclusion) connaît ces dernières années un regain d'intérêt, à l'encontre des formalismes subjectivistes, et même par exemple, à côté du réalisme néo-thomiste et aristotélicien, la philosophie de la nature de Hegel, dont Largeault aurait pu rappeler qu'elle s'inspirait parfois des critiques pertinentes de Lagrange contre le newtonianisme.

Cet ouvrage étincelant et nullement disparate, en dépit de quelques apparences contraires, souffre de quelques défauts de forme : à mon sens bien orienté (malgré les ambiguïtés du « réalisme »), utilement et spirituellement critique sans politesses superflues, il risque de décourager souvent son lecteur en lui laissant le soin de vérifier difficultueusement ce que trop souvent l'auteur présente par esquisses et allusions, en des matières où il est facile à presque tout le monde de perdre pied. Selon le mot de Terrasson popularisé par Kant, c'eût été plus court (à lire) si c'eût été plus long (en volume). Une certaine précipitation de l'auteur rejoint peut-être le souci de l'éditeur, mais il se pourrait que l'erreur fût double : on se trompe en se refusant à être pédagogiquement accessible et on se trompe encore quand on trouve qu'une réflexion de philosophie des sciences venant d'une compétence reconnue peut et doit se limiter à un cercle d'initiés sans aspirer à « une bonne vente ". La grande qualité et la nouveauté de ces Principes mériteraient une explicitation plus large et plus aisée ${ }^{4}$. À l'homme d'affaires de dire : Soyez brefs. Disons : Sachons être longs, quand cela en vaut la peine.

Jean BERNHARDT.

4. La pire des solutions : intercaler les chapitres et leurs notes (qui, justement sont d'une très grande richesse). Et comment deviner que par ex. MMM est le sigle de l'ouvrage de R. THOM : Méthodes mathématiques de la morphogenèse? 